\title{
Hepatic transcript profiling in early-lactation dairy cows fed rumen-protected niacin during the transition from late pregnancy to lactation
}

\author{
R. Ringseis, ${ }^{1 *}$ J. O. Zeitz, ${ }^{1}$ A. Weber, ${ }^{1}$ C. Koch, ${ }^{2}$ and K. Eder ${ }^{1}$ \\ ${ }^{1}$ Institute of Animal Nutrition and Nutrition Physiology, Justus-Liebig-University Giessen, Heinrich-Buff-Ring 26-32, 35392 Giessen, Germany \\ ${ }^{2}$ Educational and Research Centre for Animal Husbandry, Hofgut Neumuehle, 67728 Muenchweiler an der Alsenz, Germany
}

\begin{abstract}
In dairy cows, administration of high dosages of niacin (nicotinic acid, NA) was found to cause antilipolytic effects, which are mediated by the NA receptor hydroxyl-carboxylic acid receptor 2 (HCAR2) in white adipose tissue (WAT), and thereby an altered hepatic lipid metabolism. However, almost no attention has been paid to possible direct effects of NA in cattle liver, despite evidence that HCAR2 is also expressed in the liver and is even more abundant than in WAT. Because of this, we hypothesized that feeding a high dosage of rumen-protected NA to dairy cows influences critical metabolic or signaling pathways in the liver by inducing changes in the hepatic transcriptome. To identify these pathways, we applied genome-wide transcript profiling in liver biopsies obtained at d 7 postpartum (p.p.) from dairy cows used in our recent study; cows received either no NA (control group, $\mathrm{n}=9$ ) or $79 \mathrm{mg}$ of rumenprotected NA $/ \mathrm{kg}$ of body weight daily (NA group, $\mathrm{n}$ $=9$ ) from $21 \mathrm{~d}$ before calving until 3 wk p.p. Hepatic transcript profiling revealed that 487 transcripts were differentially expressed (filter criteria: fold change $>1.2$ or $<-1.2$ and $P<0.05$ ) in the liver at d 7 p.p. between cows fed NA and control cows. Substantially more transcripts were downregulated $(\mathrm{n}=338)$, whereas only 149 transcripts were upregulated by NA in the liver of cows. Gene set enrichment analysis for the upregulated transcripts revealed that the most-enriched gene ontology biological process terms were exclusively related to immune processes, such as leukocyte differentiation, immune system process, activation of immune response, and acute inflammatory response. Gene set enrichment analysis of the downregulated transcripts showed that the most-enriched biological process terms
\end{abstract}

Received June 18, 2018.

Accepted September 13, 2018.

*Corresponding author: robert.ringseis@ernaehrung.uni-giessen.de were related to metabolic processes, such as cellular metabolic process, small molecule metabolic process, lipid catabolic process, organic cyclic compound metabolic process, small molecule biosynthetic process, and cellular lipid catabolic process. In conclusion, hepatic transcriptome analysis showed that rumen-protected NA induces genes that are involved mainly in immune processes, including acute phase response and stress response, in dairy cows at d 7 p.p. Thus, supplementation of a high dosage of rumen-protected NA to dairy cows in the periparturient period may induce or amplify the systemic inflammation-like condition that is typically observed in the liver of high-yielding dairy cows in the p.p. period.

Key words: niacin, liver, lactating dairy cow, acute phase response

\section{INTRODUCTION}

Niacin or nicotinic acid (NA) is a water-soluble vitamin that is provided to animals from different dietary sources and in ruminants from microbial synthesis in the rumen (Brent and Bartley, 1984). It is required in the animal's tissues as a precursor of nicotinamide adenine dinucleotide $\left(\mathrm{NAD}^{+}\right)$and nicotinamide adenine dinucleotide phosphate $\left(\mathrm{NADP}^{+}\right)$, which are important coenzymes in numerous enzymatic reactions in intermediary metabolism. Apart from serving as a precursor for coenzymes, NA at pharmacologic dosages exerts pronounced plasma lipid-modulating effects, such as decreasing triacylglycerols (TAG) and very low density lipoproteins (VLDL); indeed, NA has been in clinical use as an effective drug for the treatment of different forms of dyslipidemia in humans for more than 5 decades (Carlson, 2005). The plasma lipid-modulating effects of NA have been largely ascribed to its antilipolytic effect, which is mediated by activation of the white adipose tissue (WAT) NA receptor hydroxyl-carboxylic acid receptor 2 (HCAR2), thereby decreasing plasma levels of nonesterified fatty acids (NEFA) and availability of NEFA for TAG synthesis and VLDL assembly in the liver (Gille et al., 2008). 
In dairy cows, administration of high dosages of NA either in rumen-protected form or via the abomasum to reduce or avoid ruminal degradation of NA was also found to cause antilipolytic effects (Pires et al., 2007; Pires and Grummer, 2007; Pescara et al., 2010; Morey et al., 2011; Yuan et al., 2012). Such effects might be helpful in high-yielding dairy cows during the postpartum (p.p.) period because dairy cows are typically in strong negative energy balance during this phase, leading to an excessive mobilization of NEFA from WAT into the circulation. High circulating levels of NEFA, together with a hepatic inflammation-like condition typically arising in the p.p. period in high-yielding dairy cows (Bradford et al., 2015), are considered major challenges for the liver, predisposing p.p. dairy cows to the development of hepatic lipid-related disorders such as fatty liver and ketosis. In line with this, 2 studies reported at least a tendency for reduced liver TAG concentrations in dairy cows supplemented with rumen-protected NA in the p.p. period (Morey et al., 2011; Yuan et al., 2012). In addition, it was shown that rumen-protected NA (Morey et al., 2011) and even unprotected NA (Erickson et al., 1992; Fronk and Schultz, 1979) decrease the plasma concentration of BHB in p.p. dairy cows. We have also recently demonstrated that plasma concentrations of BHB in dairy cows fed a TMR without (control) or with rumen-protected NA from $21 \mathrm{~d}$ before calving until 3 wk p.p. were decreased in the early p.p. period (Zeitz et al., 2018). However, in our study, rumen-protected NA failed to lower plasma concentrations of NEFA and liver concentrations of TAG (Zeitz et al., 2018). This indicates that the antilipolytic effect of NA mediated via HCAR2 in WAT alone cannot convincingly explain the reduced plasma levels of BHB; additional effects of NA in other tissues might be involved. In this regard, it is noteworthy that NA receptor HCAR2 is also expressed in the liver of cattle, where it is even more abundant than in WAT (Titgemeyer et al., 2011), whereas in humans and mice HCAR2 mRNA is absent in the liver and predominantly found in WAT and immune cells (Tunaru et al., 2003, Wise et al., 2003). Moreover, HCAR2 expression in the liver of cattle was found not only in tissue-resident immune cells of the liver (Kupffer cells), but also in parenchymal cells (Titgemeyer et al., 2011). This suggests that NA in cattle may cause direct effects in the liver that are mediated independently of its antilipolytic effects. Because of the abundant HCAR2 expression in cattle liver, we hypothesized that feeding rumen-protected NA to dairy cows influences critical metabolic or signaling pathways in the liver by inducing changes in the hepatic transcriptome. To identify these pathways, we applied genome-wide transcript profiling in liver biopsies obtained at d 7 p.p. from dairy cows that received either no NA (control group) or a daily rumen-protected NA dosage (NA group) from $21 \mathrm{~d}$ before calving until 3 wk p.p.

\section{MATERIALS AND METHODS}

\section{Animal Experiment}

A total of 22 multiparous Holstein dairy cows were used, which were part of a study carried out at the Educational and Research Centre for Animal Husbandry, Hofgut Neumühle (Münchweiler an der Alsenz, Germany) and approved by the Provincial Government of Coblenz, Germany (23 177-07/G 14-20-050). The experimental period lasted from 3 wk before expected calving until 1 wk p.p. Details on feeding and husbandry conditions are given in Zeitz et al. (2018). In brief, cows were assigned to 2 groups (control group and NA group, each $n=11$ ) and kept in a freestall barn equipped with weighing troughs. Clinical symptoms of infectious and other diseases that were treated by a veterinarian were found in 3 cows of the control group (1 with mastitis, 1 with anorexia and diarrhea, 1 with milk fever) and 3 cows of the NA group (1 with metritis, 1 with mastitis, 1 with milk fever). The cases of mastitis were treated locally with cefquinom (Cobactan, MSD Tiergesundheit, Unterschleißheim, Germany), whereas the cows with metritis were treated with tetracycline (Bela-Pharm, Vechta, Germany). Cows with milk fever and anorexia and diarrhea received an infusion therapy. Both groups of cows received the same corn and grass silage-based antepartum and p.p. TMR during pregnancy and lactation, respectively. The diets were calculated to meet the energy and protein requirements of dairy cows following the recommendations of the German Society of Nutrition Physiology (GfE, 2001). The cows had free access to the TMR, which was supplied twice daily at 0700 and $1400 \mathrm{~h}$. In addition, cows of the NA group were given $79 \mathrm{mg}$ of rumen-protected NA (NiaShure; Balchem, New Hampton, NY) per kg of BW daily. Considering the rumen degradability of NiaShure (Zeitz et al., 2018), the applied NA dosage was equivalent to $47 \mathrm{mg}$ of NA at the duodenum and estimated to be equivalent to $31 \mathrm{mg}$ of absorbable NA per $\mathrm{kg}$ of $\mathrm{BW}$ based on information given by the supplier. This NA dosage was close to that required for pharmacological use as an antilipidemic drug in humans (10-30 mg of NA/kg of BW; Offermanns, 2006). The NA dosage was adapted to the BW of the cows once per week starting 3 wk before expected calving until 1 wk p.p. The individual NA dosage was mixed into a 500-g pressed sugar beet pulp portion, which was supplied to each cow once 
daily at $0800 \mathrm{~h}$ in feed fences, ensuring complete intake. Cows of the control group were given $500 \mathrm{~g}$ of pressed sugar beet pulp without supplemented NA.

\section{Sample Collection}

Blood samples were collected on d 7 p.p. between 0700 and $0800 \mathrm{~h}$ (after milking and before feeding) from tail vein into EDTA-containing vacutainers (S-Monovette $9 \mathrm{~mL}$ K3E, Sarstedt, Nümbrecht, Germany) and kept on ice for $<1 \mathrm{~h}$. Plasma was obtained by centrifugation at $2,000 \times g$ for $10 \mathrm{~min}$ at $4^{\circ} \mathrm{C}$ and stored at $-20^{\circ} \mathrm{C}$. Biopsies of the liver were also taken on d 7 p.p. at 0900 $\mathrm{h}$ between the 9 th and 10th ribs approximately $15 \mathrm{~cm}$ below the transverse processes by means of a self-made barbed 12-gauge $\times 27 \mathrm{~cm}$ biopsy needle under local anesthesia, as described in detail (Zeitz et al., 2018). Liver samples were immediately snap-frozen in liquid $\mathrm{N}_{2}$ and stored at $-80^{\circ} \mathrm{C}$.

\section{RNA Isolation}

Total RNA isolation from liver samples was carried out using the RNeasy Mini Kit (Qiagen, Hilden, Germany) according to the manufacturer's protocol and stored at $-80^{\circ} \mathrm{C}$. The concentration and purity of the RNA was estimated from the optical density at 260 and $280 \mathrm{~nm}$, respectively, using a NanoQuant plate (Infinite 200, Tecan, Mainz, Germany). Before sample processing at the Centre of Excellence for Fluorescent Bioanalytics (University of Regensburg, Regensburg, Germany), the concentration and integrity of RNA was analyzed using an Agilent 2100 Bioanalyzer (Agilent Technologies, Böblingen, Germany). The total RNA concentration, optical density A260:A280 ratios, and RNA integrity number (RIN) values of all samples were $0.22 \pm 0.08 \mu \mathrm{g} / \mu \mathrm{L}, 2.00 \pm 0.03$, and $7.2 \pm 0.5$ (mean \pm $\mathrm{SD}, \mathrm{n}=18$ ), respectively. From the 11 available RNA samples per group, the 2 samples with the lowest RIN values were excluded.

\section{Microarray Hybridization and Analysis}

For microarray analysis, 9 total RNA samples per group were used. Total RNA samples were processed according to the GeneChip WT Plus Reagent Kit (Affymetrix, High Wycombe, UK) as recently described in detail (Gessner et al., 2017). The Bovine Gene 1.0 Sense Target array (Affymetrix), representing approximately 23,000 bovine transcripts, was used. The microarray data have been submitted in MIAME (minimum information about a microarray experiment)-compliant format to the National Center for Biotechnology Information (NCBI) Gene Expression Omnibus (GEO) public repository (Edgar et al., 2002; GEO accession no. GSE116058). Microarray analysis was carried out using the Robust Multichip Analysis (RMA) algorithm (Irizarry et al., 2003). Annotation of microarrays was performed with the Affymetrix BovGene-1_0-stv1_Probeset_Release 36 annotation file. Transcripts were defined as differentially expressed when the fold change (FC) between the NA group and control group was $>1.2$ or $<-1.2$ and the $P$-value of the unpaired Student's $t$-test (2-tailed distribution, 2 -sample equal variance) for each transcript was $<0.05$. Filtering of differentially expressed transcripts using the GEO2R tool (http://www.ncbi.nlm.nih.gov/geo/geo2r/) with Benjamini and Hochberg false discovery rate adjustment method failed, because the adjusted $P$-values for all transcripts were $>0.05$.

\section{Bioinformatic Prediction of mRNA Targets of the Differentially Expressed miRNAs}

The mRNA targets of differentially regulated microRNAs (miRNA) were predicted in silico using TargetScan release version 7.1 (http://www.targetscan .org/) based on the presence of conserved 6 to $8 \mathrm{mer}$ sites matching the seed region of each miRNA (Lewis et al., 2005). A cumulative weighted "context++" score, which is a measure of predicted efficacy of targeting of the sites (Agarwal et al., 2015), of $<-0.50$ was used as cut-off criterion for predicting mRNA targets.

\section{Gene Set Enrichment Analysis}

Gene set enrichment analysis (GSEA) using gene ontology (GO) terms within GO categories (biological process, cellular component, molecular function) was applied to extract biological meaning from the identified differentially expressed transcripts and predicted mRNA targets; GO terms with a $P$-value $<0.05$ were considered as enriched. The GSEA was carried out using the Database for Annotation, Visualization and Integrated Discovery (DAVID) 6.8 bioinformatic resource (Huang et al., 2009a,b).

\section{Quantitative PCR Analysis}

Quantitative PCR (qPCR) analysis in liver tissue was carried out as described in our recent paper (Zeitz et al., 2018). Gene-specific primer pairs for the reference genes were the same as used by Zeitz et al. (2018). Primer pairs for target genes were designed using PRIMER3 and BLAST and synthesized by Eurofins MWG Operon (Ebersberg, Germany). Primer characteristics are shown in Supplemental Table S1 (https:// doi.org/10.3168/jds.2018-15232). 


\section{Statistical Analysis}

All data were evaluated by Student's $t$-test using the Minitab statistical software (release 13, Minitab Inc., State College, PA).

\section{RESULTS}

\section{Performance of Dairy Cows}

Feed intake, milk and milk protein yields, milk urea concentration, $\mathrm{BW}, \mathrm{BCS}$, and the extent of negative energy balance did not differ between cows fed rumenprotected NA and control cows at wk 1 to 3 p.p., as reported recently (Zeitz et al., 2018). Milk fat percentage and milk fat yield at wk 1 to 3 of lactation were lower in cows fed rumen-protected NA than in control cows (Zeitz et al., 2018).

\section{Identification of NA-Regulated Transcripts in the Liver}

To investigate the effect of NA on the hepatic transcriptome of early-lactating dairy cows, we used a bovine microarray representing approximately 23,000 Bos taurus transcripts. Taking into account the filter criteria, 487 transcripts were found to be differentially expressed in the liver between cows fed NA and control cows. Only 149 transcripts were upregulated by NA in the liver of cows; substantially more transcripts were downregulated $(\mathrm{n}=338)$. The 30 most strongly up- and downregulated transcripts are presented in Table 1 and Table 2, respectively. Although all of the upregulated transcripts were protein-coding mRNAs, the downregulated transcripts consisted of 319 proteincoding mRNAs and 19 non-protein-coding miRNAs. The latter are summarized in Table 3.

\section{Validation of Microarray Data for Selected NA-Regulated mRNAs by qPCR}

Microarray data were validated by $\mathrm{qPCR}$ analysis of 12 differentially regulated mRNAs, which were randomly selected from the most strongly up- and downregulated mRNAs. Table 4 shows that in the case of all mRNAs to be validated, the effect direction was the same between qPCR and microarray data. Because

Table 1. The 30 most strongly upregulated transcripts in the liver of cows fed rumen-protected niacin (NA) compared with control cows at 1 wk postpartum

\begin{tabular}{|c|c|c|}
\hline Gene symbol & Gene description & $\begin{array}{l}\text { Fold change }{ }^{1} \\
\text { NA vs. control }\end{array}$ \\
\hline$H P$ & Haptoglobin & 2.85 \\
\hline ECEL1 & Endothelin converting enzyme-like 1 & 2.67 \\
\hline NNAT & Neuronatin & 2.40 \\
\hline S100A12 & S100 calcium binding protein A12 & 1.92 \\
\hline LOC782061 & Aldo-keto reductase family 1 , member C1-like & 1.89 \\
\hline LOC100296421 & UDP-glucuronosyltransferase 2B4 & 1.74 \\
\hline FOLR3 & Folate receptor 3 (gamma) & 1.66 \\
\hline C5H12orf75 & Chromosome 5 open reading frame, human c12orf75 & 1.65 \\
\hline$B O L A-D Q A 5$ & Major histocompatibility complex, class II, DQ $\propto 5$ & 1.57 \\
\hline$A P C S$ & Amyloid P component, serum & 1.56 \\
\hline TRGC6 & T-cell receptor gamma chain TRGC6 & 1.50 \\
\hline CD28 & CD28 molecule & 1.48 \\
\hline SPATA9 & Spermatogenesis associated 9 & 1.47 \\
\hline$G N M T$ & Glycine $N$-methyltransferase & 1.45 \\
\hline C2H2orf88 & Chromosome 2 open reading frame, human c2orf 88 & 1.42 \\
\hline TYROBP & TYRO protein tyrosine kinase binding protein & 1.41 \\
\hline$N C A L D$ & Neurocalcin delta & 1.41 \\
\hline LOC789690 & Olfactory receptor 12D2 & 1.39 \\
\hline LOC613345 & H3 histone, family 3A-like & 1.39 \\
\hline MAP2 & Microtubule-associated protein 2 & 1.38 \\
\hline LOC100335322 & $60 \mathrm{~S}$ ribosomal protein L22-like 1 & 1.37 \\
\hline$G P X_{4}$ & Glutathione peroxidase 4 & 1.36 \\
\hline LOC511657 & Olfactory receptor 8B3 & 1.36 \\
\hline C20H5orf49 & Chromosome 20 open reading frame, human c5orf 49 & 1.35 \\
\hline$S R X N 1$ & Sulfiredoxin 1 & 1.35 \\
\hline TGM5 & Transglutaminase 5 & 1.35 \\
\hline CIAPIN1 & Cytokine induced apoptosis inhibitor 1 & 1.34 \\
\hline$T R D$ & T-cell receptor delta chain & 1.32 \\
\hline LY86 & Lymphocyte antigen 86 & 1.32 \\
\hline$A L D O A$ & Aldolase A, fructose-bisphosphate & 1.32 \\
\hline
\end{tabular}

${ }^{1}$ Fold change was calculated from the signal $\log$ ratios as follows: $2^{\text {Signal log ratio }}$ if signal $\log$ ratio $\geq 0$ and $(-1)$ $\times 2^{\text {-(Signal } \log \text { ratio) }}$ if signal $\log$ ratio $<0$. Signal $\log$ ratios were calculated from $\mathrm{n}=9$ microarrays per group. 
Table 2. The 30 most strongly downregulated transcripts in the liver of cows fed rumen-protected niacin (NA) compared with control cows at 1 wk postpartum

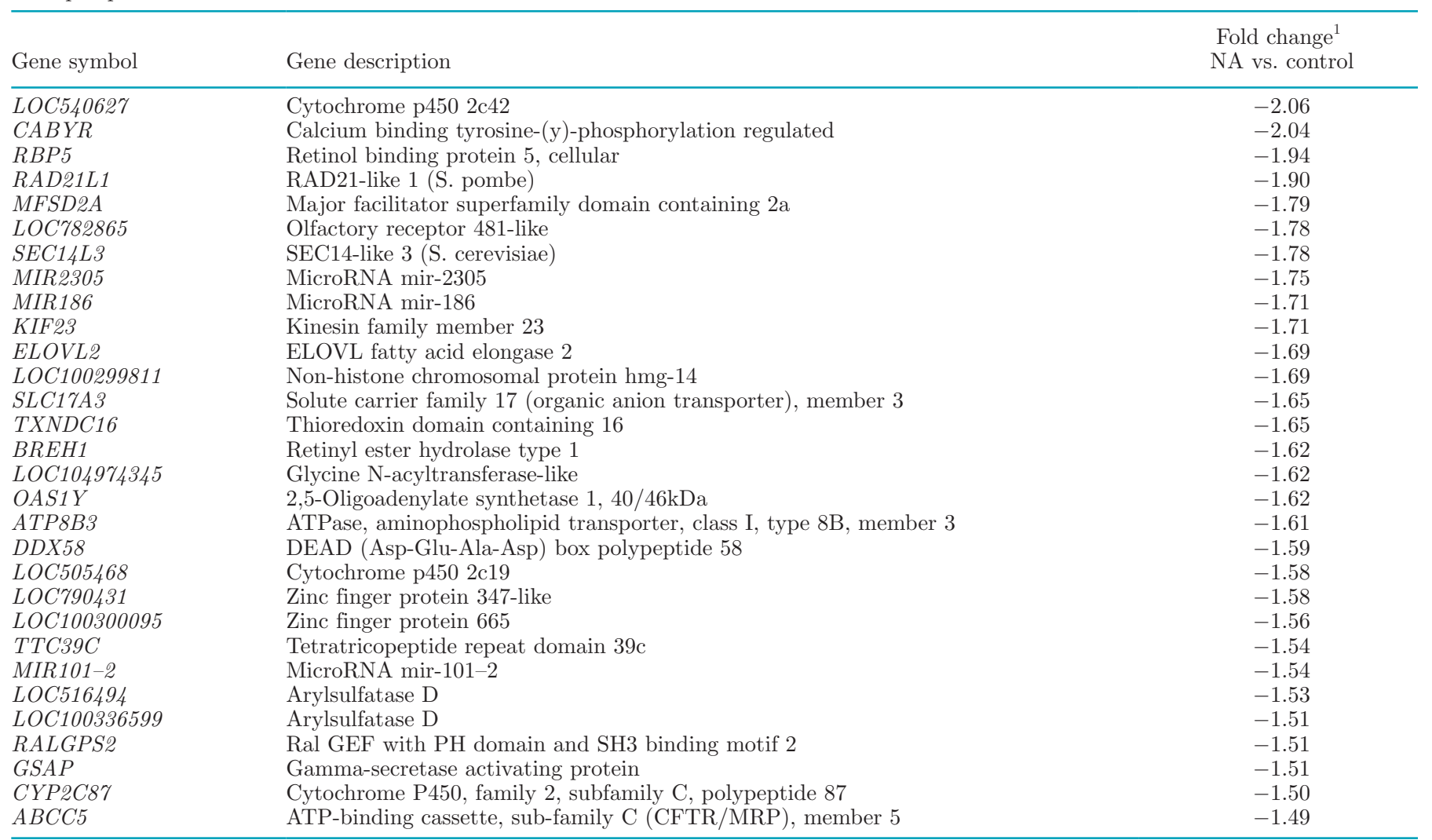

${ }^{1}$ Fold change was calculated from the signal $\log$ ratios as follows: $2^{\text {Signal } \log \text { ratio }}$ if signal $\log$ ratio $\geq 0$ and $(-1) \times 2^{- \text {(Signal } \log \text { ratio) }}$ if signal $\log$ ratio $<0$. Signal $\log$ ratios were calculated from $n=9$ microarrays per group.

Table 3. MicroRNAs downregulated in the liver of cows fed rumenprotected niacin (NA) compared with control cows at 1 wk postpartum

\begin{tabular}{llc}
\hline $\begin{array}{l}\text { Gene } \\
\text { symbol }\end{array}$ & Gene description & $\begin{array}{c}\text { Fold change } \\
\text { NA vs. } \\
\text { control }\end{array}$ \\
\hline MIR2305 & MicroRNA mir-2305 & -1.75 \\
MIR186 & MicroRNA mir-186 & -1.71 \\
MIR101-2 & MicroRNA mir-101-2 & -1.54 \\
MIR30F & MicroRNA mir-30f & -1.41 \\
MIR2475 & MicroRNA mir-2475 & -1.41 \\
MIR23B & MicroRNA mir-23b & -1.38 \\
MIR2303 & MicroRNA mir-2303 & -1.37 \\
MIR2483 & MicroRNA mir-2483 & -1.37 \\
MIR27B & MicroRNA mir-27b & -1.36 \\
MIR2461 & MicroRNA mir-2461 & -1.36 \\
MIR2465 & MicroRNA mir-2465 & -1.35 \\
MIR33B & MicroRNA mir-33b & -1.33 \\
MIR103-2 & MicroRNA mir-103-2 & -1.33 \\
MIR2473 & MicroRNA mir-2473 & -1.31 \\
MIR365-2 & MicroRNA mir-365-2 & -1.30 \\
MIR664A & MicroRNA mir-664a & -1.25 \\
MIR23A & MicroRNA mir-23a & -1.24 \\
MIR660 & MicroRNA mir-660 & -1.22 \\
MIR2291 & MicroRNA mir-2291 & -1.22 \\
\hline
\end{tabular}

${ }^{1}$ Fold change was calculated from the signal $\log$ ratios as follows: $2^{\text {Signal } \log \text { ratio }}$ if signal $\log$ ratio $\geq 0$ and $(-1) \times 2^{- \text {(Signal } \log \text { ratio) }}$ if signal $\log$ ratio $<0$. Signal $\log$ ratios were calculated from $n=9$ microarrays per group. of the different number of biological replicates and the different detection principle of transcript abundance between microarray and $\mathrm{qPCR}$, the FC and $P$-values differed more or less. Statistical analysis revealed that 5 of the 12 mRNAs analyzed by qPCR were regulated by NA treatment at a significance level of $P<0.05$ (HP, TYROBP, RBP5, MFSDQA, BREH1) and 4 at a significance level of $P<0.1$ (FOLR3, LOC540627, ATP8B3, DDX58), whereas 3 mRNAs were not differentially regulated (APCS, GNMT, LOC505468).

\section{Identification of Biological Processes Affected by NA-Regulated Protein-Coding Transcripts in the Liver}

To identify biological processes affected by the NAregulated protein-coding transcripts, GSEA was performed using GO biological process terms. The GSEA of the upregulated mRNAs revealed that the mostenriched biological process terms (lowest $P$-value) were exclusively related to immune processes. Examples of the most-enriched terms are regulation of leukocyte differentiation, immune system process, leukocyte dif- 
Table 4. Real-time quantitative (q)PCR validation of selected microarray data

\begin{tabular}{|c|c|c|c|c|}
\hline \multirow[b]{2}{*}{ Gene symbol } & \multicolumn{2}{|c|}{ Fold change } & \multicolumn{2}{|c|}{$P$-value } \\
\hline & Microarray $^{1}$ & $\mathrm{qPCR}^{2}$ & Microarray & qPCR \\
\hline$H P$ & 2.85 & 6.05 & 0.032 & 0.044 \\
\hline FOLR3 & 1.66 & 1.58 & 0.011 & 0.082 \\
\hline$A P C S$ & 1.56 & 1.68 & 0.046 & 0.158 \\
\hline GNMT & 1.45 & 1.72 & 0.042 & 0.200 \\
\hline TYROBP & 1.41 & 1.81 & 0.012 & 0.036 \\
\hline LOC540627 (CYP2C42) & -2.06 & -1.85 & 0.014 & 0.073 \\
\hline$R B P 5$ & -1.94 & -2.87 & 0.001 & 0.024 \\
\hline$M F S D 2 A$ & -1.79 & -1.73 & 0.031 & 0.007 \\
\hline BREH1 & -1.62 & -1.49 & 0.003 & 0.042 \\
\hline АТР $8 B 3$ & -1.61 & -1.90 & 0.020 & 0.064 \\
\hline$D D X 58$ & -1.59 & -1.90 & 0.023 & 0.051 \\
\hline LOC505468 (CYP2C19) & -1.58 & -1.54 & 0.045 & 0.292 \\
\hline
\end{tabular}

ferentiation, hematopoiesis, immune system development, regulation of immune system process, activation of immune response, and acute inflammatory response. Table 5 shows all biological process terms with $P$-values $<0.01$, including the number of genes assigned to these terms.

The GSEA of the downregulated mRNAs showed that the most-enriched biological process terms were related to metabolic processes, such as cellular metabolic process, small molecule metabolic process, lipid catabolic process, organic cyclic compound metabolic process, small molecule biosynthetic process, and cellular lipid catabolic process. Table 6 shows all biological process terms with $P$-values $<0.01$, including the number of genes assigned to these terms.

\section{Identification of Cellular Components of Proteins Encoded by the NA-Regulated Protein-Coding Transcripts in the Liver}

To further identify cellular components of proteins encoded by the NA-regulated protein-coding transcripts, GSEA was performed using GO cellular component terms separately for the up- and downregulated mRNAs. The GSEA of the upregulated mRNAs showed that the most-enriched cellular component terms were blood microparticle, extracellular exosome, and extracellular vesicle. All cellular component terms with $P$ values $<0.01$ including the number of genes assigned to these terms, are listed in Table 7.

The GSEA of the downregulated mRNAs showed that the most enriched cellular component terms were dealing with lipoprotein particles: chylomicron, triglyceride-rich lipoprotein particle, and VLDL particle. All cellular component terms with $P$-values $<0.01$, includ- ing the number of genes assigned to these terms, are listed in Table 8.

\section{Prediction of Target mRNAs of the NA-Regulated miRNAs and Functional Analysis}

To identify further protein-coding transcripts that are regulated by rumen-protected NA in the liver of cows, we performed bioinformatic target mRNA prediction for the top 10 regulated miRNAs. Considering a cumulative weighted context ++ score $<-0.50,831 \mathrm{mRNA}$ targets were identified. Data including gene names, total and 8 mer, 7 mer, and 6mer sites and cumulative weighted context++ score are shown in Supplemental Table S2 (https://doi.org/10.3168/jds.2018-15232). To clarify the biological relevance, we carried out GSEA using the GO category "molecular function" with the predicted mRNA targets. As shown in Table 9, enriched GO "molecular function" terms associated with the mRNA targets of the top 10 downregulated miRNAs were mainly involved in RNA polymerase II-dependent transcriptional activator activity.

\section{DISCUSSION}

Hepatic transcript profiling at d 7 p.p. in dairy cows fed without or with rumen-protected NA during the transition from late pregnancy to lactation revealed that approximately 490 transcripts are regulated by rumen-protected NA. Considering that only 5 transcripts were regulated $>2$-fold or $<-2$-fold indicates that the influence of rumen-protected NA on the hepatic transcriptome of p.p. dairy cows is moderate. However, we can exclude the possibility that low availability of NA from the rumen-protected NA source (NiaShure) ac- 
Table 5. Enriched gene ontology (GO) biological process terms ${ }^{1}$ associated with upregulated genes in the liver of cows fed rumen-protected niacin (NA) compared with control cows at 1 wk postpartum

\begin{tabular}{lrcc}
\hline GO biological process term & Count & $P$-value & $\begin{array}{c}\text { Fold } \\
\text { enrichment }\end{array}$ \\
\hline Regulation of leukocyte differentiation & 8 & 0.0002 & 6.38 \\
Immune system process & 25 & 0.0004 & 2.13 \\
Leukocyte differentiation & 11 & 0.0005 & 3.90 \\
Hematopoiesis & 13 & 0.0012 & 2.99 \\
Regulation of hematopoiesis & 8 & 0.0018 & 4.57 \\
Hematopoietic or lymphoid organ development & 13 & 0.0019 & 2.83 \\
Regulation of myeloid leukocyte differentiation & 5 & 0.0024 & 8.82 \\
Immune system development & 13 & 0.0029 & 2.68 \\
Regulation of immune system process & 15 & 0.0030 & 2.42 \\
Myeloid leukocyte differentiation & 6 & 0.0038 & 5.72 \\
Activation of immune response & 7 & 0.0044 & 4.51 \\
Positive regulation of immune system process & 11 & 0.0054 & 2.81 \\
Acute inflammatory response & 4 & 0.0090 & 9.25
\end{tabular}

${ }^{1}$ The GO terms are sorted by their enrichment $P$-values [Expression Analysis Systematic Explorer (EASE) score] (top: lowest $P$-value, bottom: highest $P$-value). Only GO terms with $P$-values $<0.01$ are shown.

counts for the moderate influence on hepatic transcriptome because we have recently shown (for the cows of this study) that plasma concentration of nicotinamide, which is rapidly formed from NA in the liver, was markedly higher at $\mathrm{d} 7$ p.p. in the NA-supplemented cows than in control cows (Zeitz et al., 2018). In addition,

Table 6. Enriched gene ontology (GO) biological process terms ${ }^{1}$ associated with downregulated genes in the liver of cows fed rumen-protected niacin (NA) compared with control cows at 1 wk postpartum

\begin{tabular}{|c|c|c|c|}
\hline GO biological process term & Count & $P$-value & $\begin{array}{c}\text { Fold } \\
\text { enrichment }\end{array}$ \\
\hline Cellular metabolic process & 129 & 0.0001 & 1.26 \\
\hline Small molecule metabolic process & 36 & 0.0001 & 1.98 \\
\hline Metabolic process & 134 & 0.0006 & 1.20 \\
\hline Lipid catabolic process & 11 & 0.0009 & 3.65 \\
\hline Organic cyclic compound metabolic process & 78 & 0.0013 & 1.36 \\
\hline Small molecule biosynthetic process & 13 & 0.0023 & 2.82 \\
\hline Single-organism biosynthetic process & 24 & 0.0023 & 1.97 \\
\hline Cellular lipid catabolic process & 8 & 0.0025 & 4.31 \\
\hline Triglyceride catabolic process & 4 & 0.0028 & 13.86 \\
\hline Single-organism catabolic process & 17 & 0.0033 & 2.27 \\
\hline Positive regulation of lipoprotein lipase activity & 3 & 0.0037 & 31.18 \\
\hline Positive regulation of triglyceride catabolic process & 3 & 0.0037 & 31.18 \\
\hline Catabolic process & 32 & 0.0038 & 1.69 \\
\hline Cellular catabolic process & 27 & 0.0047 & 1.77 \\
\hline Positive regulation of triglyceride lipase activity & 3 & 0.0049 & 27.28 \\
\hline Monocarboxylic acid metabolic process & 13 & 0.0049 & 2.56 \\
\hline Neutral lipid catabolic process & 4 & 0.0052 & 11.19 \\
\hline Acylglycerol catabolic process & 4 & 0.0052 & 11.19 \\
\hline Plasma lipoprotein particle organization & 4 & 0.0052 & 11.19 \\
\hline Primary metabolic process & 119 & 0.0058 & 1.18 \\
\hline Fatty acid metabolic process & 10 & 0.0058 & 3.03 \\
\hline Lipid transport & 9 & 0.0059 & 3.31 \\
\hline Protein-lipid complex subunit organization & 4 & 0.0064 & 10.39 \\
\hline Glycerolipid catabolic process & 4 & 0.0071 & 10.04 \\
\hline Organic substance catabolic process & 30 & 0.0075 & 1.65 \\
\hline Organic substance metabolic process & 124 & 0.0076 & 1.16 \\
\hline Steroid esterification & 3 & 0.0078 & 21.83 \\
\hline Cholesterol esterification & 3 & 0.0078 & 21.83 \\
\hline Sterol esterification & 3 & 0.0078 & 21.83 \\
\hline Regulation of triglyceride catabolic process & 3 & 0.0078 & 21.83 \\
\hline Single-organism metabolic process & 53 & 0.0083 & 1.39 \\
\hline Cellular aromatic compound metabolic process & 72 & 0.0094 & 1.29 \\
\hline Positive regulation of fatty acid biosynthetic process & 3 & 0.0094 & 19.84 \\
\hline Regulation of lipoprotein lipase activity & 3 & 0.0094 & 19.84 \\
\hline
\end{tabular}

${ }^{1}$ The GO terms are sorted by their enrichment $P$-values [Expression Analysis Systematic Explorer (EASE) score] (top: lowest $P$-value, bottom: highest $P$-value). Only GO terms with $P$-values $<0.01$ are shown. 
Table 7. Enriched gene ontology (GO) cellular component terms ${ }^{1}$ associated with upregulated genes in the liver of cows fed rumen-protected niacin (NA) compared with control cows at 1 wk postpartum

\begin{tabular}{lccc}
\hline GO cellular component term & Count & $P$-value & $\begin{array}{c}\text { Fold } \\
\text { enrichment }\end{array}$ \\
\hline Blood microparticle & 6 & 0.00014 & 11.97 \\
Extracellular exosome & 29 & 0.00022 & 2.03 \\
Extracellular vesicle & 29 & 0.00024 & 2.02 \\
Extracellular organelle & 29 & 0.00024 & 2.02 \\
Membrane-bounded vesicle & 33 & 0.00027 & 1.88 \\
Extracellular space & 18 & 0.00036 & 2.64 \\
Vesicle & 33 & 0.00056 & 1.81 \\
Extracellular region & 37 & 0.00105 & 1.66 \\
Extracellular region part & 34 & 0.00108 & 1.72 \\
Cell surface & 10 & 0.00754 & 2.88 \\
Collagen trimer & 4 & 0.00998 & 8.92 \\
19he GO terms are sorted by their enrichment $P$-values [Expression Analysis Systematic Explorer (EASE) \\
score] (top: lowest $P$-value, bottom: highest $P$-value). Only GO terms with $P$-values $<0.01$ are shown.
\end{tabular}

the plasma nicotinamide concentration at $\mathrm{d} 7$ p.p. in NA-supplemented cows was significantly higher than in most other studies with dairy cows fed either rumenprotected NA (Morey et al., 2011; Yuan et al., 2012) or unprotected NA (Niehoff et al., 2009). The marked elevation of plasma nicotinamide is probably the result of the high daily NA dosage provided to the cows of the NA group, which was markedly higher than in the abovementioned studies (Niehoff et al., 2009; Morey et al., 2011; Yuan et al., 2012). Despite the overall moderate influence on hepatic gene expression, a significant finding of this study was that genes upregulated by rumen-protected NA were almost exclusively involved in immune processes. In fact, the most-enriched biological process terms related to upregulated genes were regulation of leukocyte differentiation, immune system process, immune system development, regulation of immune system process, activation of immune response, and acute inflammatory response. The involvement of the upregulated genes in immune processes was reflected by the identification of numerous genes dealing with immune processes among the list of upregulated genes such as HP, NNAT, S100A12, BOLA-DQA5, APCS, TRGC6, CD28, NCALD, GPX4, SRXN1, CIAPIN1, and $T R D$. Of note, among these genes, we identified 2 acute phase proteins (APP) - HP (the most strongly upregulated gene in the liver of NA-treated dairy cows) and $A P C S$ - suggesting that rumen-protected NA induces or amplifies (considering that a systemic inflammation-like condition typically occurs in the liver of high-yielding dairy cows in the p.p. period) an acute phase response in the liver of dairy cows in the early p.p. period. This assumption was substantiated by our observation that induction of hepatic HP in NA-treated dairy cows could be confirmed by qPCR. Because cows with clinical symptoms of infectious (mastitis, metritis) and noninfectious diseases (milk fever), which are known to induce an acute phase response, were simi-

Table 8. Enriched gene ontology (GO) cellular component terms ${ }^{1}$ associated with downregulated genes in the liver of cows fed rumen-protected niacin (NA) compared with control cows at 1 wk postpartum

\begin{tabular}{lrcc}
\hline GO cellular component term & Count & $P$-value & $\begin{array}{c}\text { Fold } \\
\text { enrichment }\end{array}$ \\
\hline Chylomicron & 4 & 0.0002 & 31.8 \\
Triglyceride-rich lipoprotein particle & 4 & 0.0007 & 22.0 \\
Very low density lipoprotein particle & 4 & 0.0007 & 22.0 \\
RNA polymerase I transcription factor complex & 3 & 0.0011 & 53.7 \\
Intracellular & 173 & 0.0019 & 1.1 \\
Nucleoplasm & 42 & 0.0019 & 1.6 \\
Nuclear lumen & 52 & 0.0041 & 1.5 \\
Intracellular membrane-bounded organelle & 134 & 0.0053 & 1.2 \\
Plasma lipoprotein particle & 4 & 0.0054 & 11.0 \\
Lipoprotein particle & 4 & 0.0054 & 11.0 \\
Protein-lipid complex & 4 & 0.0067 & 10.2 \\
Intracellular part & 164 & 0.0081 & 1.1 \\
Intracellular organelle lumen & 55 & 0.0086 & 1.4 \\
Organelle lumen & 55 & 0.0087 & 1.4 \\
1The GO terms are sorted by their enrichment $P$-values TExpression Analysis Systematic Explorer $($ EASE) \\
score] (top: lowest $P$-value, bottom: highest $P$-value). Only GO terms with $P$-values $<0.01$ are shown.
\end{tabular}


Table 9. Enriched gene ontology (GO) molecular function terms ${ }^{1}$ associated with the target mRNAs of the top 10 downregulated microRNAs in the liver of cows fed rumen-protected niacin (NA) compared with control cows at 1 wk postpartum

\begin{tabular}{|c|c|c|c|}
\hline GO molecular function term & Count & $P$-value & $\begin{array}{c}\text { Fold } \\
\text { enrichment }\end{array}$ \\
\hline RNA polymerase II transcription factor activity, sequence-specific DNA binding & 52 & 0.000004 & 1.99 \\
\hline Protein binding & 474 & 0.000057 & 1.11 \\
\hline Transcription factor activity, sequence-specific DNA binding & 76 & 0.000105 & 1.56 \\
\hline Nucleic acid binding transcription factor activity & 76 & 0.000108 & 1.56 \\
\hline Sequence-specific DNA binding & 67 & 0.000255 & 1.57 \\
\hline Sequence-specific double-stranded DNA binding & 49 & 0.000318 & 1.71 \\
\hline Binding & 598 & 0.000367 & 1.05 \\
\hline Protein kinase regulator activity & 18 & 0.000468 & 2.65 \\
\hline RNA polymerase II regulatory region DNA binding & 42 & 0.000537 & 1.76 \\
\hline Macromolecular complex binding & 76 & 0.000604 & 1.47 \\
\hline Transcription regulatory region sequence-specific DNA binding & 46 & 0.000714 & 1.68 \\
\hline
\end{tabular}

${ }^{1}$ The GO terms are sorted by their enrichment $P$-values [Expression Analysis Systematic Explorer (EASE) score] (top: lowest $P$-value, bottom: highest $P$-value). Only GO terms with $P$-values $<0.001$ are shown.

larly distributed among the 2 groups, we are confident that the effect of NA supplementation was not biased by the illness history of the cows. However, future studies should investigate whether our observation from transcriptome analysis of an amplification of an acute phase response in dairy cows fed rumen-protected NA is accompanied by increased plasma concentrations of APP. In the present study, we did not consider the plasma concentrations of APP, because APP, like many other blood parameters, are known to exhibit marked time-dependent fluctuations in dairy cows, which might have biased the effect of NA supplementation, and the plasma concentrations of APP respond to an inflammatory or stress signal with a certain time shift. In addition, future studies are needed to clarify whether our observation at the transcriptome level can be attributed to the relatively high daily NA dosage.

Apart from induction of APP-encoding genes, an interesting finding was that several stress-responsive genes were identified as upregulated, such as antioxidant proteins (SRXN1, GPX4), calcium binding proteins (S100A12, NCALD) and drug-metabolizing/ detoxifying enzymes (AKR1C1,UGT2B4), genes that are known to be regulated by the stress-responsive transcription factors nuclear factor- $\kappa \mathrm{B}(\mathrm{NF}-\kappa \mathrm{B})$ and nuclear factor (erythroid-derived 2)-like 2 (Nrf2). This observation indicates that rumen-protected NA induces a stress-response in the liver of p.p. dairy cows. A further indication of the initiation of stress response in the liver of cows fed rumen-protected NA is the identification of ATF3 and E2F4 as upregulated genes. The ATF3 protein belongs to the ATF/CREB family of transcription factors (Hai and Hartman, 2001), encoded by an immediate-early gene that is rapidly induced by a wide spectrum of stress signals (Hai et al., 1999). As a transcription factor, ATF3 directly or indirectly alters the expression of a variety of genes, and one common characteristic for many of its target genes is their ability to regulate inflammation (Hai et al., 2010). The E2F4 gene is also a stress-responsive gene (Crosby et al., 2007), encoding a member of the family of E2F transcription factors that play a key role in the $\mathrm{G}_{1} / \mathrm{S}$ cell cycle checkpoint and the control of cell proliferation (Bertoli et al., 2013). Interestingly, members of the E2F transcription factors regulate inflammatory signaling by physically interacting with NF- $\kappa \mathrm{B}$ (Ankers et al., 2016). The exact reason for the induction of a stress response in the liver of cows fed rumen-protected NA is unclear. However, a stress response is typically initiated to cope with the presence of stress-inducing stimuli. Important stress-inducing stimuli, which are known to activate redox-sensitive transcription factors such as NF- $\kappa \mathrm{B}$ and Nrf2, are reactive oxygen species (ROS). At least in studies with rats, it was shown that NA supplementation increases hydrogen peroxide levels in plasma (Sun et al., 2012; Li et al., 2013a) and causes a dosage-dependent increase in oxidative DNA damage in the liver ( $\mathrm{Li}$ et al., 2013b). In addition, a study with Jurkat cells demonstrated that NA stimulates ROS production in vitro (Crowley et al., 2000). In dairy cows, at least indirect evidence for the stimulation of ROS production by NA supplemented during the periparturient period was provided in a recent study showing that the ferric-reducing ability of plasma was decreased, indicating a decreased plasma antioxidative capacity in cows at 2 wk p.p. (Bühler et al., 2018b). Bühler et al. (2018b) attributed the effect to an improved energy status of NA-supplemented cows due to higher 
$\mathrm{NAD}^{+}$and $\mathrm{NADP}^{+}$levels, leading to an increased cell respiration and activity of ROS-generating superoxide dismutase. One might speculate that the NA-induced oxidative stress in p.p. dairy cows is responsible for the observation that the number of early-apoptotic polymorphonuclear leukocytes and late-apoptotic peripheral blood mononuclear cells is increased in dairy cows receiving supplemental NA during the periparturient period (Bühler et al., 2016), because oxidative stress is known to stimulate different signaling pathways in immune cells, leading to apoptotic cell death (Wedi et al., 1999). In any case, the findings from Bühler et al. (2016) and those from another study from Bühler et al. (2018a), in which the phagocytotic activity of blood immune cells was investigated, show that NA supplementation in periparturient dairy cows affects immune function in the p.p. period.

With regard to the genes downregulated in cows fed rumen-protected NA, these genes were mainly involved in different metabolic processes, such as small molecule metabolic process, lipid catabolic process, organic cyclic compound metabolic process, small molecule biosynthetic process, and cellular lipid catabolic process. This was reflected by the identification of many genes encoding metabolic enzymes and proteins, such as CYP2C42, RBP5, ELOVL2, SLC17A3, ATP8B3, BREH1, and CYP2C19. The induction of genes encoding certain enzymes of the cytochrome P450 family (CYP2C42, CYP2C19) may indicate that drug metabolism and catabolism of steroid hormones is influenced by NA supplementation in the liver of cows in the early p.p. period. For instance, progesterone is inactivated primarily in the liver of cows by different CYP enzymes, including those of the $2 \mathrm{C}$ subfamily (Murray, 1991, 1992). In line with this, it was found that feeding dairy cows a high-cornstarch diet reduces hepatic activities of CYP2C and CYP3A and increases progesterone half-life compared with feeding a highfiber diet (Lemley et al., 2010). From the literature, no data are available to demonstrate that rumen-protected NA influences circulating levels of progesterone. However, future studies should address this issue because increased hepatic progesterone catabolism decreases circulating levels of progesterone, and insufficient circulating progesterone levels have been implicated as a major cause of poor fertility in lactating dairy cows (Lonergan, 2011). It is also interesting that several genes encoding apolipoproteins, such as apolipoprotein $(\boldsymbol{A P O}) A 1, A P O A 5, A P O B$, and APOC2, were among the genes downregulated by rumen-protected NA. As important constituents of lipoproteins, apolipoproteins are formed in the liver to be assembled with hepatic lipids, such as TAG and phospholipids, and are secreted as VLDL particles into the circulation (Shelness and
Sellers, 2001). Our observation from transcript profiling therefore suggests that rumen-protected NA affects hepatic lipoprotein assembly and secretion in dairy cows in the early p.p. period. In line with this, bioinformatic analysis of downregulated genes revealed that the most enriched cellular component terms were chylomicron, triglyceride-rich lipoprotein particle, and VLDL particle. Impaired hepatic production of apolipoproteins is in agreement with the above-described indications of induction of an acute phase response in the liver of cows fed rumen-protected NA. It is well known that in the course of the acute phase response, the liver increases the production of so-called positive APP, such as HP, APCS, and many others, which play major roles in restoring tissue homeostasis during the early phase of inflammation, injury, trauma, and infection (Venteclef et al., 2011; Ceciliani et al., 2012). In contrast, the production of several other proteins normally synthesized by the liver at high rates, such apolipoproteins, $\mathrm{RBP}$, transferrin, and albumin, is reduced during the acute phase response; these proteins are called negative APP. Consequently, the pool of free amino acids can be preferentially utilized for the production of positive APP and de novo synthesis of glucose (Ceciliani et al., 2012). This means that immune function has priority over anabolic function. Despite indications from the hepatic transcriptome signature that rumen-protected NA impairs hepatic lipoprotein assembly and secretion in dairy cows, no evidence for this has been found at the phenotypic level. In fact, in our recently published study, we showed that TAG concentration in the liver did not differ between NA-supplemented and control cows at 1 and 3 wk p.p. (Zeitz et al., 2018). Also, the TAG concentration in plasma of these cows did not differ between NA-supplemented and control cows in the p.p. period (Zeitz et al., 2018). One explanation for this discrepancy between hepatic transcriptome signature and phenotype may be that the effects of rumen-protected NA on hepatic gene expression were only moderate overall. For instance, the genes encoding apolipoproteins (APOA1, APOA5, APOB, APOC2) were downregulated in the NA-treated cows by only -1.45 - to -1.21 -fold. Obviously, this effect is not biologically relevant, at least in this specific case. This clearly indicates that data from transcript profiling may provide valuable information about metabolic and signaling pathways affected by a specific treatment. However, transcriptomic data must be cautiously interpreted and it is important to confirm, by determining suitable phenotypic parameters, whether these changes in gene expression are of biological significance.

A further interesting finding of the present study was that rumen-protected NA causes downregulation of several miRNAs in the liver of dairy cows. MicroRNAs 
are small non-protein-coding RNAs that regulate gene expression mainly at the posttranscriptional level by binding to complementary mRNA sequences, leading to degradation of the specific mRNAs or repression of protein translation (Hutvágner and Zamore, 2002). Interestingly, a single miRNA can regulate hundreds of protein-encoding target mRNAs, indicating the great regulatory potential of miRNAs (Bartel, 2004). We recently demonstrated in a study with rats that NA at a pharmacologic dosage alters the expression of miRNAs in skeletal muscle, and in silico target gene prediction indicated that more than 1,800 mRNAs are putative targets of NA-regulated miRNAs (Couturier et al., 2014). Moreover, we showed that many of the predicted target mRNAs from the most strongly downregulated miRNAs are involved in transcription regulator activity and regulation of transcription from RNA polymerase, indicating that processes dealing with gene transcription are activated by NA treatment because the mRNAs from downregulated miRNAs are less targeted and, thus, less degraded. Interestingly, the recent rat study also showed that several stressresponsive transcription factors including ATF3, ATF1, and one member of the E2F family, E2F 7 , are targets of the miRNAs downregulated by NA (Couturier et al., 2014). In the liver of cows fed rumen-protected NA, 831 mRNAs were predicted as targets of the 10 most strongly downregulated miRNAs. As in skeletal muscle of rats, we found, in the liver of cows, that processes dealing with gene transcription (e.g., RNA polymerase II transcription factor activity, sequence-specific DNA binding, transcriptional activator activity) are the most enriched molecular function terms associated with these miRNA-regulated mRNA targets. In addition, as in skeletal muscle of rats, the stress-sensitive transcription factor E2F7 could be identified as a target of the miRNAs downregulated by rumen-protected NA in the liver of cows. Thus, based on these similar findings between rats and dairy cows, we propose that miRNA-mRNA interactions are generally involved in NA-dependent regulation of gene expression and that these interactions may play a specific role in the activation of the stress response in the liver of p.p. dairy cows.

\section{CONCLUSIONS}

Hepatic transcriptome analysis showed that rumenprotected NA induces genes that are involved mainly in immune processes, including acute phase response and stress response in dairy cows at $\mathrm{d} 7 \mathrm{p}$.p. These findings indicate that supplementation of rumen-protected NA to dairy cows in the periparturient period may induce or amplify the systemic inflammation-like condition that is typically observed in the liver of high-yielding dairy cows in the p.p. period. Because the daily dosage of NA was markedly higher than in recent studies dealing with rumen-protected NA, future studies have to clarify whether the relatively high NA dosage is responsible for the indications of an inflammation-like condition.

\section{ACKNOWLEDGMENTS}

This study was supported by a grant from the German Research Foundation (DFG, Bonn, Germany), project no. ED 70/13-1. We gratefully acknowledge the staff of the Educational and Research Centre for Animal Husbandry, Hofgut Neumuehle (Muenchweiler an der Alsenz, Germany) and the technical staff of the University Giessen (Giessen, Germany).

\section{REFERENCES}

Agarwal, V., G. W. Bell, J. W. Nam, and D. P. Bartel. 2015. Predicting effective microRNA target sites in mammalian mRNAs. eLife 4:e05005. https://doi.org/10.7554/eLife.05005.

Ankers, J. M., R. Awais, N. A. Jones, J. Boyd, S. Ryan, A. D. Adamson, C. V. Harper, L. Bridge, D. G. Spiller, D. A. Jackson, P. Paszek, V. Sée, and M. R. White. 2016. Dynamic NF-кB and E2F interactions control the priority and timing of inflammatory signalling and cell proliferation. eLife 5:e10473.

Bartel, D. P. 2004. MicroRNAs: Genomics, biogenesis, mechanism, and function. Cell 116:281-297.

Bertoli, C., S. Klier, C. McGowan, C. Wittenberg, and R. A. de Bruin. 2013. Chk1 inhibits E2F6 repressor function in response to replication stress to maintain cell-cycle transcription. Curr. Biol. 23:1629-1637.

Bradford, B. J., K. Yuan, J. K. Farney, L. K. Mamedova, and A. J. Carpenter. 2015. Invited review: Inflammation during the transition to lactation: New adventures with an old flame. J. Dairy Sci. 98:6631-6650.

Brent, B. E., and E. E. Bartley. 1984. Thiamin and niacin in the rumen. J. Anim. Sci. 59:813-822.

Bühler, S., J. Frahm, W. Liermann, R. Tienken, S. Kersten, U. Meyer, K. Huber, and S. Dänicke. 2018a. Effects of energy supply and nicotinic acid supplementation on phagocytosis and ROS production of blood immune cells of periparturient primi- and pluriparous dairy cows. Res. Vet. Sci. 116:62-71.

Bühler, S., J. Frahm, R. Tienken, S. Kersten, U. Meyer, K. Huber, and S. Dänicke. 2016. Influence of energy level and nicotinic acid supplementation on apoptosis of blood leukocytes of periparturient dairy cows. Vet. Immunol. Immunopathol. 179:36-45.

Bühler, S., J. Frahm, R. Tienken, S. Kersten, U. Meyer, K. Huber, and S. Dänicke. 2018b. Effects of energy supply and nicotinic acid supplementation on serum anti-oxidative capacity and on expression of oxidative stress-related genes in blood leucocytes of periparturient primi- and pluriparous dairy cows. J. Anim. Physiol. Anim. Nutr. (Berl.) 102:e87-e98.

Carlson, L. A. 2005. Nicotinic acid: The broad-spectrum lipid drug. A 50th anniversary review. J. Intern. Med. 258:94-114.

Ceciliani, F., J. J. Ceron, P. D. Eckersall, and H. Sauerwein. 2012. Acute phase proteins in ruminants. J. Proteomics 75:4207-4231.

Couturier, A., J. Keller, E. Most, R. Ringseis, and K. Eder. 2014. Niacin in pharmacological doses alters microRNA expression in skeletal muscle of obese Zucker rats. PLoS One 9:e98313.

Crosby, M. E., J. Jacobberger, D. Gupta, R. M. Macklis, and A. Almasan. 2007. E2F4 regulates a stable G2 arrest response to genotoxic stress in prostate carcinoma. Oncogene 26:1897-1909.

Crowley, C. L., C. M. Payne, H. Bernstein, C. Bernstein, and D. Roe. 2000. The NAD+ precursors, nicotinic acid and nicotinamide pro- 
tect cells against apoptosis induced by a multiple stress inducer, deoxycholate. Cell Death Differ. 7:314-326.

Edgar, R., M. Domrachev, and A. E. Lash. 2002. Gene Expression Omnibus: NCBI gene expression and hybridization array data repository. Nucleic Acids Res. 30:207-210.

Erickson, P. S., M. R. Murphy, and J. H. Clark. 1992. Supplementation of dairy cow diets with calcium salts of long-chain fatty acids and nicotinic acid in early lactation. J. Dairy Sci. 75:1078-1089.

Fronk, T. J., and L. H. Schultz. 1979. Oral nicotinic acid as a treatment for ketosis. J. Dairy Sci. 62:1804-1807.

Gessner, D. K., A. Winkler, C. Koch, G. Dusel, G. Liebisch, R. Ringseis, and K. Eder. 2017. Analysis of hepatic transcript profile and plasma lipid profile in early lactating dairy cows fed grape seed and grape marc meal extract. BMC Genomics 18:253.

GfE (German Society of Nutrition Physiology). 2001. Recommendations for energy and nutrient requirements for dairy cows and heifers. DLG-Verlag, Frankfurt a. Main, Germany. [in German].

Gille, A., E. T. Bodor, K. Ahmed, and S. Offermanns. 2008. Nicotinic acid: pharmacological effects and mechanisms of action. Annu. Rev. Pharmacol. Toxicol. 48:79-106.

Hai, T., and M. G. Hartman. 2001. The molecular biology and nomenclature of the ATF/CREB family of transcription factors: ATF proteins and homeostasis. Gene 273:1-11.

Hai, T., C. D. Wolfgang, D. K. Marsee, A. E. Allen, and U. Sivaprasad. 1999. ATF3 and stress responses. Gene Expr. 7:321-335.

Hai, T., C. C. Wolford, and Y. S. Chang. 2010. ATF3, a hub of the cellular adaptive-response network, in the pathogenesis of diseases: Is modulation of inflammation a unifying component? Gene Expr. 15:1-11.

Huang, D. W., B. T. Sherman, and R. A. Lempicki. 2009a. Systematic and integrative analysis of large gene lists using DAVID bioinformatics resources. Nat. Protoc. 4:44-57.

Huang, D. W., B. T. Sherman, and R. A. Lempicki. 2009b. Bioinformatics enrichment tools: Paths toward the comprehensive functional analysis of large gene lists. Nucleic Acids Res. 37:1-13.

Hutvágner, G., and P. D. Zamore. 2002. A microRNA in a multipleturnover RNAi enzyme complex. Science 297:2056-2060.

Irizarry, R. A., B. Hobbs, F. Collin, Y. D. Beazer-Barclay, K. J. Antonellis, U. Scherf, and T. P. Speed. 2003. Exploration, normalization, and summaries of high density oligonucleotide array probe level data. Biostatistics 4:249-264.

Lemley, C. O., T. A. Wilmoth, L. R. Tager, K. M. Krause, and M. E. Wilson. 2010. Effect of a high cornstarch diet on hepatic cytochrome P450 2C and 3A activity and progesterone half-life in dairy cows. J. Dairy Sci. 93:1012-1021.

Lewis, B. P., C. B. Burge, and D. P. Bartel. 2005. Conserved seed pairing, often flanked by adenosines, indicates that thousands of human genes are microRNA targets. Cell 120:15-20.

Li, D., N. Luo, Q. Ma, S. Z. Li, Q. Shi, Y. Cao, and S. S. Zhou. 2013a Excessive nicotinic acid increases methyl consumption and hydrogen peroxide generation in rats. Pharm. Biol. 51:8-12.

Li, D., Y. J. Tian, J. Guo, W. P. Sun, Y. Z. Lun, M. Guo, N. Luo, Y. Cao, J. M. Cao, X. J. Gong, and S. S. Zhou. 2013b. Nicotinamide supplementation induces detrimental metabolic and epigenetic changes in developing rats. Br. J. Nutr. 110:2156-2164.

Lonergan, P. 2011. Influence of progesterone on oocyte quality and embryo development in cows. Theriogenology 76:1594-1601.

Morey, S. D., L. K. Mamedova, D. E. Anderson, C. K. Armendariz, E. C. Titgemeyer, and B. J. Bradford. 2011. Effects of encapsulated niacin on metabolism and production of periparturient dairy cows. J. Dairy Sci. 94:5090-5104.
Murray, M. 1991. Microsomal cytochrome P450-dependent steroid metabolism in male sheep liver. Quantitative importance of 6 betahydroxylation and evidence for the involvement of a P450 from the IIIA subfamily in the pathway. J. Steroid Biochem. Mol. Biol. 38:611-619.

Murray, M. 1992. Participation of a cytochrome P450 enzyme from the $2 \mathrm{C}$ subfamily in progesterone 21-hydroxylation in sheep liver. J. Steroid Biochem. Mol. Biol. 43:591-593.

Niehoff, I. D., L. Hüther, and P. Lebzien. 2009. Niacin for dairy cattle: A review. Br. J. Nutr. 101:5-19.

Offermanns, S. 2006. The nicotinic acid receptor GPR109A (HM74A or PUMA-G) as a new therapeutic target. Trends Pharmacol. Sci. $27: 384-390$

Pescara, J. B., J. A. Pires, and R. R. Grummer. 2010. Antilipolytic and lipolytic effects of administering free or ruminally protected nicotinic acid to feed-restricted Holstein cows. J. Dairy Sci. 93:5385-5396

Pires, J. A., and R. R. Grummer. 2007. The use of nicotinic acid to induce sustained low plasma nonesterified fatty acids in feedrestricted Holstein cows. J. Dairy Sci. 90:3725-3732.

Pires, J. A., J. B. Pescara, and R. R. Grummer. 2007. Reduction of plasma NEFA concentration by nicotinic acid enhances the response to insulin in feed-restricted Holstein cows. J. Dairy Sci. 90:4635-4642.

Shelness, G. S., and J. A. Sellers. 2001. Very-low-density lipoprotein assembly and secretion. Curr. Opin. Lipidol. 12:151-157.

Sun, W. P., D. Li, Y. Z. Lun, X. J. Gong, S. X. Sun, M. Guo, L. X. Jing, L. B. Zhang, F. C. Xiao, and S. S. Zhou. 2012. Excess nicotinamide inhibits methylation-mediated degradation of catecholamines in normotensives and hypertensives. Hypertens. Res. $35: 180-185$

Titgemeyer, E. C., L. K. Mamedova, K. S. Spivey, J. K. Farney, and B. J. Bradford. 2011. An unusual distribution of the niacin receptor in cattle. J. Dairy Sci. 94:4962-4967.

Tunaru, S., J. Kero, A. Schaub, C. Wufka, A. Blaukat, K. Pfeffer, and S. Offermanns. 2003. PUMA-G and HM74 are receptors for nicotinic acid and mediate its anti-lipolytic effect. Nat. Med. 9:352-355.

Venteclef, N., T. Jakobsson, K. R. Steffensen, and E. Treuter. 2011. Metabolic nuclear receptor signaling and the inflammatory acute phase response. Trends Endocrinol. Metab. 22:333-343.

Wedi, B., J. Straede, B. Wieland, and A. Kapp. 1999. Eosinophil apoptosis is mediated by stimulators of cellular oxidative metabolisms and inhibited by antioxidants: involvement of a thiol-sensitive redox regulation in eosinophil cell death. Blood 94:2365-2373.

Wise, A., S. M. Foord, N. J. Fraser, A. A. Barnes, N. Elshourbagy, M. Eilert, D. M. Ignar, P. R. Murdock, K. Steplewski, A. Green, A. J. Brown, S. J. Dowell, P. G. Szekeres, D. G. Hassall, F. H. Marshall, S. Wilson, and N. B. Pike. 2003. Molecular identification of high and low affinity receptors for nicotinic acid. J. Biol. Chem. 278:9869-9874.

Yuan, K., R. D. Shaver, S. J. Bertics, M. Espineira, and R. R. Grummer. 2012. Effect of rumen-protected niacin on lipid metabolism oxidative stress, and performance of transition dairy cows. J. Dairy Sci. 95:2673-2679.

Zeitz, J. O., A. Weber, E. Most, W. Windisch, C. Bolduan, J. Geyer, F. J. Romberg, C. Koch, and K. Eder. 2018. Effects of supplementing rumen-protected niacin on fiber composition and metabolism of skeletal muscle in dairy cows during early lactation. J Dairy Sci. 101:8004-8020. https://doi.org/10.3168/jds.2018-14490. 